\title{
Management and Treatment of Andersen-Tawil Syndrome (ATS)
}

\author{
Valeria Sansone* and Rabi Tawil ${ }^{\dagger}$ \\ *Department of Neurology, University of Milan, IRCCS Policlinico San Donato, Milan, Italy 20097; ${ }^{\dagger}$ Department of Neurology, \\ Strong Memorial Hospital, University of Rochester, Rochester, New York 14642-0002
}

Summary: Andersen-Tawil syndrome (ATS) is characterized by periodic paralysis, cardiac arrhythmias, and distinct facial and skeletal features. The majority of patients with ATS (ATS1) have point mutations in the $K C N J 2$ gene, which encodes the inward-rectifying potassium channel known as Kir2.1. The skeletal muscle and cardiac symptoms are accounted for, in most cases, by a dominant negative effect of the mutations on potassium channel current, resulting in prolonged depolarization of the action potential. Mechanisms of disruption of channel function include abnormal trafficking and assembly of second messengers such as phosphatidylinositol 4,5bisphosphate, abnormal gating of the channel, and incorrect folding of the Kir2.1 protein. Less apparent is the mechanism by which these mutations account for the typical facial and skeletal abnormalities. The concomitant involvement of cardiac and skeletal muscle in ATS poses unique treatment and management challenges. Because of differences in cardiac and skeletal muscle physiology, drugs that may have a beneficial effect on cardiac function may have a detrimental effect on skeletal muscle and vice versa. We review the clinical, laboratory, and genetic features of this disorder with particular emphasis on treatment and management. Key Words: Andersen-Tawil syndrome, LQT7, therapy, management, KCNJ2, ATS.

\section{INTRODUCTION}

Andersen-Tawil syndrome (ATS) is a rare periodic paralysis with distinct features and accounts for less than $10 \%$ of all periodic paralysis $(\sim 1: 500,000)$. The distinguishing features of ATS are the coexistence of abnormalities in two excitable tissues: skeletal muscle and cardiac muscle. Periodic paralysis in ATS occurs in the setting of either hyperkalemic or hypokalemia, and cardiac involvement ranges from asymptomatic ventricular arrhythmias to sudden death. The characteristic facial and skeletal abnormalities are often a diagnostic clue to ATS, although they may be subtle. ${ }^{1-4}$ Clinical heterogeneity is remarkable in this syndrome: electrocardiographic (ECG) abnormalities may be the only manifestation of an affected individual within a family with otherwise typical ATS. Recently, ATS has been linked to mutations in the KCNJ2 gene which codes for a voltagegated, inward rectifying potassium channel (Kir2.1) ${ }^{5,6}$ The majority of ATS patients described so far $(80 \%-$

Address correspondence and reprint requests to: Valeria Sansone, M.D., University of Milan, Department of Neurology, IRCCS Policlinico San Donato Hospital, Via Morandi 30, San Donato Milanese, Milan 20097 Italy; E-mail: valeria.sansone@unimi.it.
90\%) have a KCNJ2 mutation, causing paralysis and cardiac arrhythmias through a dominant-negative effect of the mutation on potassium current (ATS1). Less clear is the way these mutations account for facial and skeletal abnormalities and whether there are other clinical manifestations of ATS given the ubiquitous distribution of Kir2.1 channels.

\section{CLINICAL OBSERVATIONS}

\section{Periodic paralysis}

Episodic paralysis occurs in ATS in the setting of either hyperkalemia or hypokalemia. The initial patient described by Tawil et al. ${ }^{2}$ with potassium-sensitive periodic paralysis, did not subsequently prove to have a KCNJ2 mutation (personal communication). About 15\% of reported patients with $K C N J 2$ mutations have hyperkalemic episodes, and $20 \%$ have normokalemic episodes. The remainder of patients described with $K C N J 2$ mutations have episodes of paralysis associated with hypokalemia, indistinguishable from patients with hypokalemic periodic paralysis (HypoPP) caused by point mutations in the calcium channel gene. Triggers for the paralysis are similar to those in HypoPP, including car- 
bohydrate-rich meals and rest after exercise, and are relieved by oral potassium supplementation. During episodes of paralysis, especially with profound hypokalemia, the underlying cardiac arrhythmia may be exacerbated so careful monitoring of patients is required in these circumstances. Recovery occurs within 24 to 48 hours from the onset of an attack as is seen in HypoPP. Episodes of paralysis seem to decrease in frequency and severity with age. Interictally, proximal and distal muscle strength is usually normal. A mild degree of facial and neck flexor weakness may be observed in some patients. Myotonia does not occur in patients with ATS1 even when paralysis occurs in the setting of hyperkalemia.

\section{Cardiac manifestations}

Unlike other forms of periodic paralysis, cardiac involvement is an intrinsic feature of ATS. The first indication that ATS could be associated with long QT came from Tawil et al. ${ }^{2}$ in their first description of the syndrome. Linkage to the long QT locus on chromosome 11 was excluded. Subsequently, given the consistent finding of long QT intervals in the patients later described by Sansone et al., ${ }^{3}$ linkage to three additional long QT loci were explored and were also excluded. Ultimately, Plaster et al., 5 studying a large Mexican kindred, established linkage of ATS and identified mutations in the KCNJ2 gene. The identification of the gene led to the classification of ATS as an LQT (LQT7) syndrome in the cardiology literature. Tristani-Firouzi et al. ${ }^{6}$ subsequently characterized the functional and clinical consequences of KCNJ2 mutations associated with ATS. LQT occurs in $50 \%$ of patients with ATS. Ventricular arrhythmias occur in $84 \%$ of patients, with bidirectional ventricular tachycardia (BVT) being the most characteristic rhythm disturbance in $32 \%$ of patients. Cardiac arrest occurs in about $10 \%$ of patients. Of note, when BVT is present one has to consider ATS in the differential diagnosis of digitalis intoxication and mutations of the ryanodine receptor channel gene. Recently, in a small cohort of patients with polymorphic ventricular tachycardia (PVT) who underwent screening for mutations in the RyR2-encoded cardiac ryanodine receptor/calcium release channel and in the CASQ2-encoded calsequestrin, ${ }^{7}$ three of eight females $(40 \%)$ proved to have ATS1. None of these patients with $K C N J 2$ mutations displayed other features of ATS, illustrating the clinical heterogeneity of the syndrome and the overlap between the arrhythmic phenotype for catecholaminergic PVT and ATS1. There is still no consensus as to the possible sex-dependent effect of the KCNJ2 mutation and the likelihood of expressing BVT. Andelfinger et al. ${ }^{8}$ described a 71-member family with clinical and genetically defined ATS1 in which 13 (81\%) of 16 mutation-positive females expressed BVT compared with none present in the 25 mutation-positive males. Other studies exploring phenotypic variability concluded that no sex-dependent phenotypic differences were present in ATS1. ${ }^{9}$ In any case it should be noted that men are less prone to develop cardiac events because of shorter QT intervals as compared with women, boys, and girls. The shorter QT in men is most evident for heart rates less than $60 \mathrm{bpm}^{10}$

In the majority of patients with ATS1 (73\%) there is a prolonged terminal T-wave downslope, a wide TU wave pattern, and a biphasic and large U wave, all reflecting abnormalities in repolarization. The TU wave patterns are caused by decreased potassium inward current resulting from the KCNJ2 mutations. ${ }^{11}$ This $\mathrm{U}$ wave pattern may also be useful in distinguishing ATS1 from PVT associated with RyR2 and CASQ2 channel mutations. ${ }^{7}$ The abnormal TU wave patterns are usually absent in patients with clinical ATS not bearing $K C N J 2$ mutations. ${ }^{11}$

It is important to note that in ATS1 the occurrence of malignant ventricular tachyarrhythmias is relatively infrequent when compared with other LQT syndromes. Many ATS patients who present with frequent and complex ventricular ectopy are frequently asymptomatic. Tsuboi and Antzelevitch ${ }^{12}$ designed an experimental model of ATS using canine left ventricular wedge preparations and demonstrated that an increase in transmural dispersion of repolarization rather than QT interval is responsible for torsades de pointes in ATS1. They suggest that this mechanism may account for the lower frequency of ectopic activity in ATS1 and the less severe cardiac phenotype compared with other LQT syndromes. It is also possible that some mutations of Kir2.1 are lethal during embryogenesis and are thus self-eliminated early in fetal development.

Another intriguing aspect of ATS is that raised by Schoonderwoerd et al. ${ }^{13}$ who report a small KCNJ2 mutation-positive family with three affected individuals in whom two manifest dilated cardiomyopathy in addition to the classical features of ATS described above. Whether dilated cardiomyopathy is a primary feature of ATS or whether it is the result of long-standing ventricular arrhythmias is yet to be defined.

\section{Dysmorphic features}

The term "distinctive" skeletal features was used in the largest series of ATS patients reported initially by Sansone et al. ${ }^{3}$ because the facial abnormalities were in some cases subtle and in no way "dysmorphic." Nevertheless, dysmorphic features are present and pathognomonic of ATS. The most frequent findings, fully expressed in $71 \%$ of ATS patients, are broad forehead, hypoplastic mandible, hypotelorism, low-set ears, digit clinodactyly, and 2-3 syndactyly of the toes. Relative microcephaly is also common. Additional findings include abnormal or absence of the lateral incisors, high-arched palate, cleft 
palate, and small hands and feet. It is the presence of these traits, however subtle, which often is the clue to the diagnosis. The abnormalities are very consistent among patients, although, as in the muscle and cardiac phenotype, clinical heterogeneity is remarkable.

\section{LABORATORY INVESTIGATIONS}

The diagnosis of ATS should be considered in the differential diagnosis of any patient presenting with periodic paralysis. Patients should be questioned specifically about cardiac symptoms, such as syncope or palpitations, suggestive of arrhythmias. Special attention to the patient's physical features and careful evaluation with ECG are essential.

As in the evaluation of all periodic paralyses, secondary forms of periodic paralysis must be ruled out first with assessment of thyroid, renal, and adrenal functions. Subsequently, determination of ictal serum potassium levels is critical in the diagnostic workup. Serum potassium levels during attacks are usually reduced, with values ranging from 2.5 to $3 \mathrm{mEq} / \mathrm{L}$ in reported cases. ${ }^{2,3}$ Previously, when ictal potassium levels could not be obtained, patients were subjected to hypokalemic and hyperkalemic challenges. Such challenges should not be used for diagnostic purposes in ATS because of the risk of precipitating or worsening cardiac arrhythmias. Electrophysiologic studies are helpful in confirming the presence of skeletal muscle membrane inexcitability using the exercise nerve conduction study protocol first described by McManis et al. ${ }^{14}$ Fournier et al. ${ }^{15}$ have further refined this protocol to distinguish patients with known sodium, chloride, and calcium channel mutations according to their response to the short-exercise EMG response pattern. A positive long exercise nerve conduction study has been reported in ATS patients, ${ }^{16}$ but no ATS patients have been studied with the more elaborate Fournier protocol to see if they have a unique pattern. None of the ATS patients reported to date have had myotonia recorded on EMG.

The diagnosis of ATS is suspected if, in addition to periodic paralysis, the patient manifests at least one of the two other characteristic features of ATS. Ultimately, confirmation of the diagnosis requires genetic testing for the presence of $K C N J 2$ mutations.

\section{Genetics of ATS, animal models, and other potential clinical manifestations}

Kir2.1 channels are voltage-gated potassium channels called inward rectifiers. These comprise a family of voltage-related but not voltage-dependent channels whose function is to stabilize the membrane potential and determine the shape of the terminal portion of the action potential. ${ }^{17} \mathrm{KCNJ} 2$ encodes the inward rectifier Kir 2.1, a member of the Kir2.x subfamily, which is the critical alpha subunit of cardiac Ik1, the inward rectifier current. ${ }^{18}$ The channel is a dimer of subunits, each with two transmembrane spanning domains called M1 and M2. The $\mathrm{NH} 2$ terminal is on the $\mathrm{M} 1$ segment and the $\mathrm{COOH}$ terminal on the M2 segment. ${ }^{19}$ More than 30 point mutations in ATS have been described so far and mostly localize on the intracellular $\mathrm{COOH}$ terminal. ${ }^{5,9,20}$ Phosphatidylinositol 4,5-bisphosphate binding residues of Kir2.1 are common targets of mutations causing ATS $1 .{ }^{21}$ Most mutations have a dominant negative effect on potassium channel current. The mutation with the most potent dominant effect is the $T 75 R$ missense mutation. Other mutations act by a mechanism of haploinsufficiency, probably affecting trafficking and assembly of second messengers via the interaction of abnormal amino acid positioning along the muscle membrane where the Kir2.1 channels are localized. ${ }^{6,22-24}$

Given the ubiquitous distribution of Kir2.1 channels and the complexity of their interactions with second messengers and other associated membrane components, including anchoring and modeling molecules, it would not be surprising that the effects on these channels would extend to other molecular targets affecting other tissues or organs, either directly or indirectly. It is likely, therefore, that a wider spectrum of phenotypic expressions of $K C N J 2$ mutations exists. Sansone and colleagues (unpublished data) have found white matter hyperintense lesions in a 24-year-old woman with ATS1, which could account for a right hemiparesis and diffuse pyramidal signs found on examination. A complete neuropsychological battery of tests did not reveal any abnormalities. Further studies are in progress to better define the specificity and significance of these findings. Similarly, Yoon et al. ${ }^{25}$ performed neuropsychological tests on 10 patients from eight unrelated families and compared results with their unaffected siblings and concluded that there may be a mild dysexecutive syndrome unrelated to intelligence quotient performance. Studies on a larger number of patients will confirm or refute whether there is a neurocognitive phenotype in ATS.

\section{THERAPY AND MANAGEMENT OF ATS}

Establishment of evidence-based therapeutic interventions in ATS represents a particular challenge in ATS. Conventional randomized, controlled trials are impossible to conduct because ATS is extremely rare, making it impractical to recruit the large numbers of subjects needed for such studies. One alternative is the " $\mathrm{N}$ of 1 trial", which is a randomized placebo-controlled clinical trial in single patients and is a well established technique for use in rare diseases and in pilot studies of new drugs. ${ }^{26}$

An additional challenge is that this channelopathy affects two distinct excitable tissues, each with unique 
electrophysiologic characteristics. Consequently, drugs that may have a beneficial effect on one tissue may have a detrimental effect on the other. Treatment of patients with ATS should be closely coordinated between a neuromuscular specialist and a cardiologist with expertise in treating LQT.

\section{Treatment of periodic paralysis}

The treatment of the periodic paralysis associated with ATS aims at minimizing the frequency, severity, and duration of the paralytic episodes. This is important not only in reducing the disruptive consequences of the acute attacks on the patient's daily functions but also because it is assumed, though not proven, that recurrent uncontrolled attacks can predispose to persistent fixed weakness. Treatment involves minimizing known triggers coupled with pharmacologic agents as prophylaxis against recurrent attacks.

The carbonic anhydrase inhibitors (CAI) such as acetazolamide (250-1500 mg/day) and dichlorphenamide (50-200 mg/day), which are demonstrated to be efficacious in other forms of periodic paralysis, ${ }^{27}$ have been successfully used in ATS. ${ }^{2,3}$ Patients with sulfa allergies cannot take CAIs and about $10 \%$ of individuals develop nephrolithiasis with CAIs. For such patients, potassiumsparing diuretics such as spironolactone $(23-100 \mathrm{mg} /$ day) or triamterene $(25-100 \mathrm{mg} /$ day $)$ can be used instead. In patients with refractory attacks, CAIs can be combined with potassium sparing diuretics. In addition to potentially preventing paralytic attacks, potassium supplementation is attractive in ATS as elevated serum potassium levels shorten the QT interval, which lessens cardiac arrhythmogenicity. Potassium supplementation should be given as slow release formulations in doses not to exceed $80 \mathrm{mEq} /$ day.

\section{Treatment of cardiac arrhythmias}

Cardiac arrhythmias may be severe in ATS but there is often a discrepancy between the severity of the ECG recordings and patients' symptoms. The complex and frequent ventricular ectopy rarely degenerates into lifethreatening ventricular tachyarrhythmias and sudden death is less frequent in ATS than in other LQT syndromes. ${ }^{5-7,12}$ What ECG features, if any, predict the occurrence of symptomatic or life-threatening arrhythmias remains to be determined.

Long-term treatment is mandatory to prevent the recurrence of torsades by shortening the QTc interval. There are multiple factors in any particular individual patient that prolong the QT interval and precipitate ventricular tachycardia and torsades in patients with ATS. Such factors include sudden intense adrenergic stimulation produced by any intense emotion (grief, pain, fright, anger, fear, or startle), which can result in extrasystole followed by a long postextrasystolic pause that precipitates arrhythmia in the setting of an underlying pro- longed QT interval. Additionally, there are a number of cardiac and noncardiac medications (bepridil; phenylamine; class IA antiarrhythmic agents including quinidine, procainamide, disopyramide; class III antiarrhythmic agents including sotalol, ibutilide, azimilide, dofetilide, amiodarone; haloperidol, tricyclic antidepressants, erythromycin, grepafloxacin, moxifloxacin, and many others) that are known to prolong QT intervals. Patients should be educated about which medications and which risk-associated behaviors they should avoid to prevent precipitating cardiac symptoms.

The standard treatment options available for long-term management include the use of beta-adrenergic blockers. Propranolol is the drug most frequently used, at the daily dose of 2 to $3 \mathrm{mg} / \mathrm{kg}$, targeted toward a maximal heart rate of $130 \mathrm{bpm}$ or less on an exercise treadmill stress test. Calcium-channel blockers amlodipine and nifedipine have been used in some patients with ATS1 with success (personal observation) but studies in a large series of patients are needed to demonstrate consistency of response. Similarly, the usefulness of antiarrhythmics with sodium channel blocker function to treat ATS-associated arrhythmias has not been studied.

More aggressive treatment modalities may be needed in patients not responding to pharmacologic therapy. The use of a pacemaker and implantable defibrillator will prevent sudden cardiac death potentially arising from the torsades but will not prevent the occurrence of torsades so that beta-blockers are still recommended with this approach. Another approach that has been successful in one of our patients with ATS is left-thoracic sympathectomy. $^{2}$

\section{CONCLUSION}

ATS is a unique muscle channelopathy because it affects different excitable membranes, and in particular the cardiolemma, with a similar pathogenetic mechanism involving a member of a family of voltage-gated potassium channels called inward rectifiers (Kir2.1). The ubiquitous distribution and the role of these channels in cell membrane excitability (and also on anchoring, assembly, and trafficking of amino acids, second messengers, and other effectors) account for the complex manifestations of this syndrome. Successful treatment of ATS requires separate therapeutic interventions to treat cardiac and skeletal muscle symptoms. Current treatments modalities are based on known effective treatments in the periodic paralyses and LQT syndromes, which may not be optimal given this syndrome's unique pathophysiology. Further studies are needed to outline the optimal treatment modalities for ATS. 


\section{REFERENCES}

1. Andersen ED, Krasilnikoff PA, Overvad H. Intermittent muscular weakness, extrasystoles, and multiple developmental abnormalities: a new syndrome? Acta Pediatr Scand 1971;60:559-564.

2. Tawil R, Ptacek LJ, Pavlakis SG, et al. Andersen's syndrome: potassium-sensitive periodic paralysis, ventricular ectopy, and dysmorphic features. Ann Neurol 1994;35:326-330.

3. Sansone V, Griggs RC, Meola G, Ptacek LJ, Barohn R, Iannaccone S, Bryan W, Baker N, Janas SJ, Scott W, Ririe D, Tawil R. Andersen's syndrome: a distinct periodic paralysis. Ann Neurol 1997;42:305-312.

4. Canun S, Perez N, Beirana LG. Andersen syndrome autosomal dominant in three generations. Am J Med Genet 1999;85:147-156.

5. Plaster NM, Tawil R, Tristani-Firouzi M, et al. Mutations in Kir2.1 cause the developmental and episodic electrical phenotypes of Andersen's syndrome. Cell 2001;105:511-519.

6. Tristani-Firouzi M, Jensen JL, Donaldson MR, et al. Functional and clinical characterization of $\mathrm{KCNJ} 2$ mutations associated with LQT7 (Andersen syndrome). J Clin Invest 2002;110:381-388.

7. Tester DJ, Arya P, Will M, et al. Genotypic heterogeneity and phenotypic mimicry among unrelated patients referred for catecholaminergic polymorphic ventricular tachycardia genetic testing. Heart Rhythm 2006:3:800-805.

8. Andelfinger G, Tapper AR, Welch RC, Vanoye CG, George AL Jr, Benson DW. KCNJ2 mutation results in Andersen syndrome with sex-specific cardiac and skeletal muscle phenotypes. Am J Hum Genet 2002;71:663-668.

9. Davies NP, Imbrici P, Fialho D, et al. Andersen-Tawil syndrome: new potassium channel mutations and possible phenotypic variation. Neurology 2005;65:1083-1089.

10. Khan IA. Long QT syndrome: diagnosis and management. Am Heart J 2002;143:7-14.

11. Zhang L, Benson DW, Tristani-Firouzi M, et al. Electrocardiographic features in Andersen-Tawil syndrome patients with KCNJ2 mutations: characteristic T-U-wave patterns predict the KCNJ2 genotype. Circulation 2005;111:2720-2726.

12. Tsuboi M, Antzelevitch C. Cellular basis for electrocardiographic and arrhythmic manifestations of Andersen-Tawil syndrome (LQT7). Heart Rhythm 2006;3:328-335.

13. Schoonderwoerd BA, Wiesfeld AC, Wilde AA, et al. A family with Andersen-Tawil syndrome and dilated cardiomyopathy. Heart Rhythm 2006;3:1346-1350.
14. McManis PG, Lambert EH, Daube JR. The exercise test in periodic paralysis. Muscle Nerve 1986;9:704-710.

15. Fournier E, Arzel M, Sternberg D, et al. Electromyography guides toward subgroups of mutations in muscle channelopathies. Ann Neurol 2004;56:650-661.

16. Katz JS, Wolfe GI, Iannaccone S, Bryan WW, Barohn RJ. The exercise test in Andersen syndrome. Arch Neurol 1999;56:352356.

17. Jongsma HJ, Wilders R. Channelopathies: Kir2.1 mutations jeopardize many cell functions. Curr Biol 2001;11:R747-50.

18. Lopatin AN, Nicholas CG. Inward rectifiers in the heart: an update on Ik1. J Mol Cell Cardiol 2001;1:625-638.

19. Chen L, Kawano T, Bajic S, et al. A glutamate residue at the C-terminus regulates activity of inward rectifier $\mathrm{K}+$ channels: implication for Andersen's syndrome. Proc Natl Acad Sci U S A 2002;99:8430-8435.

20. Hosaka Y, Hanawa H, Washizuka T, et al. Function, subcellular localization and assembly of a novel mutation of $\mathrm{KCNJ} 2$ in Andersen's syndrome. J Mol Cell Cardiol 2003;35:409-415.

21. Donaldson MR, Jensen JL, Tristani-Firouzi M, et al. PIP2 binding residues of Kir2.1 are common targets of mutations causing Andersen syndrome. Neurology 2003;60:1811-1816.

22. Bendahhou S, Donaldson MR, Plaster NM, Tristani-Firouzi M, Fu YH, Ptacek LJ. Defective potassium channel Kir2.1 trafficking underlies Andersen-Tawil syndrome. J Biol Chem 2003;278:5177951785.

23. Preisig-Muller R, Schlichthorl G, Goerge T, et al. Heteromerization of Kir2.x potassium channels contributes to the phenotype of Andersen's syndrome. Proc Natl Acad Sci U S A 2002;99:77747779.

24. Donaldson MR, Yoon G, Fu YH, Ptacek LJ. Andersen-Tawil syndrome: a model of clinical variability, pleiotropy and genetic heterogeneity. Ann Med 2004;36:92-97.

25. Yoon G, Quitania L, Kramer JH, Fu YH, Miller BL, Ptacek LJ. Andersen-Tawil syndrome: definition of a neurocognitive phenotype. Neurology 2006;66:1703-1710.

26. Guyatt GH, Heyting A, Jaeschke R, Keller J, Adachi JD, Roberts RS. N of 1 randomized trials for investigating new drugs. Control Clin Trials 1990;11:88-100.

27. Tawil R, McDermott MP, Brown R Jr, et al. Randomized trials of dichlorphenamide in the periodic paralyses. Working Group on Periodic Paralysis. Ann Neurol 2000;47:46-53. 